\title{
Tensor rank is not multiplicative under the tensor product
}

\author{
Matthias Christandl ${ }^{a}$, Asger Kjærulff Jensen ${ }^{a}$, \\ Jeroen Zuiddam ${ }^{\mathrm{b}, *}$ \\ a Department of Mathematical Sciences, University of Copenhagen, \\ Universitetsparken 5, 2100 Copenhagen Ø, Denmark \\ b Centrum Wiskunde $\&$ Informatica, Science Park 123, 1098 XG Amsterdam, \\ Netherlands
}

\section{A R T I C L E I N F O}

Article history:

Received 29 August 2017

Accepted 19 December 2017

Available online 21 December 2017

Submitted by J.M. Landsberg

\section{$M S C:$}

15A69

Keywords:

Tensor rank

Border rank

Degeneration

Young flattening

Algebraic complexity theory

Quantum information theory
A B S T R A C T

The tensor rank of a tensor $t$ is the smallest number $r$ such that $t$ can be decomposed as a sum of $r$ simple tensors. Let $s$ be a $k$-tensor and let $t$ be an $\ell$-tensor. The tensor product of $s$ and $t$ is a $(k+\ell)$-tensor. Tensor rank is sub-multiplicative under the tensor product. We revisit the connection between restrictions and degenerations. A result of our study is that tensor rank is not in general multiplicative under the tensor product. This answers a question of Draisma and Saptharishi. Specifically, if a tensor $t$ has border rank strictly smaller than its rank, then the tensor rank of $t$ is not multiplicative under taking a sufficiently hight tensor product power. The "tensor Kronecker product" from algebraic complexity theory is related to our tensor product but different, namely it multiplies two $k$-tensors to get a $k$-tensor. Nonmultiplicativity of the tensor Kronecker product has been known since the work of Strassen.

It remains an open question whether border rank and asymptotic rank are multiplicative under the tensor product. Interestingly, lower bounds on border rank obtained from general-

\footnotetext{
* Corresponding author.

E-mail addresses: christandl@math.ku.dk (M. Christandl), akj@math.ku.dk (A.K. Jensen), j.zuiddam@cwi.nl (J. Zuiddam).
} 
ized flattenings (including Young flattenings) multiply under the tensor product.

() 2017 Elsevier Inc. All rights reserved.

\section{Introduction}

Let $U_{i}, V_{i}$ be finite-dimensional vector spaces over a field $\mathbb{F}$. Let $t$ be a $k$-tensor in $U_{1} \otimes \cdots \otimes U_{k}$. The tensor rank of $t$ is the smallest number $r$ such that $t$ can be written as a sum of $r$ simple tensors $u_{1} \otimes \cdots \otimes u_{k}$ in $U_{1} \otimes \cdots \otimes U_{k}$, and is denoted by $\mathrm{R}(t)$. Letting $\mathbb{F}$ be the complex numbers $\mathbb{C}$, the border rank of $t$ is the smallest number $r$ such that $t$ is a limit point (in the Euclidean topology) of a sequence of tensors in $U_{1} \otimes \cdots \otimes U_{k}$ of rank at most $r$, and is denoted by $\underline{\mathrm{R}}(t)$.

Let $t \in U_{1} \otimes \cdots \otimes U_{k}$ and $s \in V_{1} \otimes \cdots \otimes V_{\ell}$ be a $k$-tensor and an $\ell$-tensor respectively. Define the tensor product of $t$ and $s$ as the $(k+\ell)$-tensor

$$
t \otimes s \in U_{1} \otimes \cdots \otimes U_{k} \otimes V_{1} \otimes \cdots \otimes V_{\ell}
$$

If $k=\ell$, then define the tensor Kronecker product of $t$ and $s$ as the $k$-tensor

$$
t \otimes s \in\left(U_{1} \otimes V_{1}\right) \otimes \cdots \otimes\left(U_{k} \otimes V_{k}\right)
$$

obtained from $t \otimes s$ by grouping $U_{i}$ and $V_{i}$ together for each $i$. In algebraic complexity theory, the tensor Kronecker product is usually just denoted by ' $\otimes$ '. Using the tensor Kronecker product one defines the asymptotic rank of $t$ as the $\operatorname{limit}_{\lim _{n \rightarrow \infty}} \mathrm{R}\left(t^{\bigotimes n}\right)^{1 / n}$. (This limit exists and equals the infimum $\inf _{n} \mathrm{R}\left(t^{\otimes n}\right)^{1 / n}$, see for example Lemma 1.1 in [1].) Asymptotic rank is denoted by $\underset{\sim}{\mathrm{R}}(t)$.

This paper is about the relationship between tensor rank and the tensor product. It follows from the definition that rank is sub-multiplicative under the tensor product.

Proposition 1. Let $t, s$ be any tensors. Then, $\mathrm{R}(t \otimes s) \leq \mathrm{R}(t) \mathrm{R}(s)$.

The result of this paper is that the above inequality can be strict.

Theorem. Tensor rank is not in general multiplicative under tensor product. Specifically, if a tensor $t$ has border rank strictly smaller than its tensor rank, then the tensor rank of $t$ is not multiplicative under a taking a sufficiently high tensor power.

The theorem answers a question posed in the lecture notes of Jan Draisma [2, Chapter 6] and a question of Ramprasad Saptharishi (personal communication, related to an earlier version of the survey [3]). The theorem was stated as a fact in [4, page 1097], referring to [5] for the proof; however, [5] studies only the tensor Kronecker product $\otimes$. 
It has been known since the work of Strassen that tensor rank is not multiplicative under the tensor Kronecker product $\otimes$, see Example 3.

We construct three instances of this phenomenon (Proposition 13, Proposition 17 and Proposition 18) to prove the theorem. Explicitly, one of our examples is the following strict inequality (Proposition 14).

Example 2. Let $b_{1}, b_{2}$ be the standard basis of $\mathbb{C}^{2}$. Define the 3 -tensor $W_{3}$ as $b_{2} \otimes b_{1} \otimes$ $b_{1}+b_{1} \otimes b_{2} \otimes b_{1}+b_{1} \otimes b_{1} \otimes b_{2} \in\left(\mathbb{C}^{2}\right)^{\otimes 3}$. Then we have the strict inequality $\mathrm{R}\left(W_{3}^{\otimes 2}\right) \leq$ $8<9=\mathrm{R}\left(W_{3}\right)^{2}$.

In Section 5 we will prove that Example 2 is essentially minimal over the complex numbers, in the sense that if $s \in \mathbb{C} \otimes \mathbb{C}^{2} \otimes \mathbb{C}^{2}$ and $t \in \mathbb{C}^{2} \otimes \mathbb{C}^{n} \otimes \mathbb{C}^{m}$, then one has $\mathrm{R}(s \otimes t)=\mathrm{R}(s \otimes t)=\mathrm{R}(s) \mathrm{R}(t)$. This we prove using the theory of canonical forms of matrix pencils and a formula for their tensor rank.

Our general approach is to study approximate decompositions (or border rank decompositions) of tensors. It turns out that a border rank decomposition of a tensor $t$ can be transformed into a tensor rank decomposition of tensor powers of $t$ with a penalty that depends on the so-called error degree of the approximation. More precisely, the notion of border rank $\underline{\mathrm{R}}(t)$ has a more precise variant $\mathrm{R}^{e}(t)$ that allows only approximations with error degree at most $e$ (see Section 2 for definitions). This variant goes back to [6] and [7]. We prove in Corollary 11(1) that

$$
\mathrm{R}\left(s^{\otimes n}\right) \leq(n e+1) \mathrm{R}^{e}(s)^{n},
$$

which we use to construct nonmultiplicativity examples. In particular, we see that as soon as $\mathrm{R}^{e}(s)<\mathrm{R}(s)$, the quantity $\mathrm{R}(s)^{n}$ grows faster than the right-hand side of (1) and thus leads to nonmultiplicativity examples for large enough $n$.

It follows from the definitions that also border rank and asymptotic rank are submultiplicative under the tensor product: $\underline{\mathrm{R}}(t \otimes s) \leq \underline{\mathrm{R}}(t) \underline{\mathrm{R}}(s)$, and $\underset{\sim}{\mathrm{R}}(t \otimes s) \leq \underset{\sim}{\mathrm{R}}(t) \underset{\sim}{\mathrm{R}}(s)$. We leave it as an open question whether these inequalities can be strict. In Section 4 we will see that lower bounds on border rank obtained from generalized flattenings (including Young flattenings) are in fact multiplicative under the tensor product.

It follows from $\mathrm{R}(t \otimes s) \leq \mathrm{R}(t \otimes s)$ that tensor rank, border rank and asymptotic rank are submultiplicative under the tensor Kronecker product: $\mathrm{R}(t \otimes s) \leq \mathrm{R}(t) \mathrm{R}(s)$, $\underline{\mathrm{R}}(t \nabla s) \leq \underline{\mathrm{R}}(t) \underline{\mathrm{R}}(s)$, and $\underset{\sim}{\mathrm{R}}(t \nabla s) \leq \underset{\sim}{\mathrm{R}}(t) \underset{\sim}{\mathrm{R}}(s)$. If $t$ and $s$ are 2-tensors (matrices), then tensor rank, border rank and asymptotic rank are equal and multiplicative under the tensor Kronecker product. However, for $k \geq 3$, it is well-known that each of the three inequalities can be strict, see the following example.

Example 3. Consider the following tensors

$$
\mathrm{T}(\circlearrowright)=\sum_{i \in\{1,2\}} b_{i} \otimes b_{i} \otimes 1 \in \mathbb{F}^{2} \otimes \mathbb{F}^{2} \otimes \mathbb{F}
$$




$$
\begin{aligned}
& \mathrm{T}\left(\prod^{\bullet}\right)=\sum_{i \in\{1,2\}} b_{i} \otimes 1 \otimes b_{i} \in \mathbb{F}^{2} \otimes \mathbb{F} \otimes \mathbb{F}^{2} \\
& \mathrm{~T}(\bullet)=\sum_{i \in\{1,2\}} 1 \otimes b_{i} \otimes b_{i} \in \mathbb{F} \otimes \mathbb{F}^{2} \otimes \mathbb{F}^{2} .
\end{aligned}
$$

(This graphical notation is borrowed from [8].) Each tensor has rank, border rank and asymptotic rank equal to 2 , since they are essentially identity matrices. However the tensor Kronecker product is the $2 \times 2$ matrix multiplication tensor

$$
\langle 2,2,2\rangle=\mathrm{T}(\Longrightarrow)=\sum_{i, j, k \in\{1,2\}}\left(b_{i} \otimes b_{j}\right) \otimes\left(b_{j} \otimes b_{k}\right) \otimes\left(b_{k} \otimes b_{i}\right)
$$

whose tensor rank and border rank is at most 7 [9] and whose asymptotic rank is thus at most 7 , which is strictly less that $2^{3}=8$. (The tensor rank of $\langle 2,2,2\rangle$ equals 7 over any field [10] and the border rank of $\langle 2,2,2\rangle$ equals 7 over the complex numbers $\mathbb{C}[11]$. Both statements are in fact true for any tensor with the same support as $\langle 2,2,2\rangle[12]$.)

\section{Degeneration and restriction}

We revisit the theory of degenerations and restrictions of tensors and how to transform degenerations into restrictions. Our non-multiplicativity results rely on these ideas. Let $t \in U_{1} \otimes \cdots \otimes U_{k}$ and $s \in V_{1} \otimes \cdots \otimes V_{k}$ be $k$-tensors. We say $t$ restricts to $s$, written $t \geq s$, if there are linear maps $A_{i}: U_{i} \rightarrow V_{i}$ such that $\left(A_{1} \otimes \cdots \otimes A_{k}\right) t=s$. Let $d, e \in \mathbb{N}$. We say $t$ degenerates to $s$ with approximation degree $d$ and error degree $e$, written $t \unrhd_{d}^{e} s$, if there are linear maps $A_{i}(\varepsilon): U_{i} \rightarrow V_{i}$ depending polynomially on $\varepsilon$ such that $\left(A_{1}(\varepsilon) \otimes \cdots \otimes A_{k}(\varepsilon)\right) t=\varepsilon^{d} s+\varepsilon^{d+1} s_{1}+\cdots+\varepsilon^{d+e} s_{e}$ for some tensors $s_{1}, \ldots, s_{e}$. Naturally, $t \unrhd^{e} s$ means $\exists d: t \unrhd_{d}^{e} s$, and $t \unrhd_{d} s$ means $\exists e: t \unrhd_{d}^{e} s$, and $t \unrhd s$ means $\exists d \exists e: t \unrhd_{d}^{e} s$. (We note that our notation $t \unrhd_{d} s$ corresponds to $t \unrhd_{d+1} s$ in [5].) Clearly, degeneration is multiplicative in the following sense.

Proposition 4. Let $t_{1}, t_{2}, s_{1}, s_{2}$ be tensors. If $t_{1} \unrhd_{d_{1}}^{e_{1}} s_{1}$ and $t_{2} \unrhd_{d_{2}}^{e_{2}} s_{2}$, then $t_{1} \otimes t_{2} \unrhd_{d_{1}+d_{2}}^{e_{1}+e_{2}}$ $s_{1} \otimes s_{2}$ and $t_{1} \otimes t_{2} \unrhd_{d_{1}+d_{2}}^{e_{1}+e_{2}} s_{1} \otimes s_{2}$.

The error degree $e$ is upper bounded by the approximation degree $d$ in the following way.

Proposition 5. Let $t, s$ be $k$-tensors. If $t \unrhd_{d} s$, then $t \unrhd_{d}^{k d-d} s$.

Proof. Suppose $\left(A_{1}(\varepsilon) \otimes \cdots \otimes A_{k}(\varepsilon)\right) t=\varepsilon^{d} s+\varepsilon^{d+1} s_{1}+\cdots+\varepsilon^{d+e} s_{e}$. For every $i$ let $B_{i}(\varepsilon)$ be the matrix obtained from $A_{i}(\varepsilon)$ by truncating each entry in $A_{i}(\varepsilon)$ to degree at most $d$. Then $\left(B_{1}(\varepsilon) \otimes \cdots \otimes B_{k}(\varepsilon)\right) t=\varepsilon^{d} s+\varepsilon^{d+1} u_{1}+\cdots+\varepsilon^{k d} u_{k d}$ for some $k$-tensors $u_{1}, \ldots, u_{k d}$. 
For any $r \in \mathbb{N}$, let $b_{1}, \ldots b_{r}$ denote the standard basis of $\mathbb{F}^{r}$. Let $r, k \in \mathbb{N}$ and let

$$
\mathrm{T}_{r}(k):=\sum_{i=1}^{r}\left(b_{i}\right)^{\otimes k} \in\left(\mathbb{F}^{r}\right)^{\otimes k}
$$

be the rank-r order- $k$ unit tensor. Let $s \in V_{1} \otimes \cdots \otimes V_{k}$. The tensor rank of $s$ is the smallest number $r$ such that $\mathrm{T}_{r}(k) \geq s$, and is denoted by $\mathrm{R}(s)$. This definition of tensor rank is easily seen to be equivalent to the definition given in the introduction. The border rank of $s$ is the smallest number $r$ such that $\mathrm{T}_{r}(k) \unrhd s$, and is denoted by $\underline{\mathrm{R}}(s)$. Note that this definition works over any field $\mathbb{F}$. When $\mathbb{F}$ equals $\mathbb{C}$, this definition of border rank is equivalent to the definition given in the introduction $[13-15,5]$. Define

$$
\begin{aligned}
& \mathrm{R}_{d}^{e}(s):=\min \left\{r \in \mathbb{N} \mid \mathrm{T}_{r}(k) \unrhd_{d}^{e} s\right\} \\
& \mathrm{R}_{d}(s):=\min \left\{r \in \mathbb{N} \mid \mathrm{T}_{r}(k) \unrhd_{d} s\right\} \\
& \mathrm{R}^{e}(s):=\min \left\{r \in \mathbb{N} \mid \mathrm{T}_{r}(k) \unrhd^{e} s\right\} .
\end{aligned}
$$

(Our notation $\mathrm{R}_{d}(s)$ corresponds to $\mathrm{R}_{d+1}(s)$ in [5].) Error degree in the context of border rank was already studied in [6] and [7]. The following propositions follow directly from Proposition 4 and Proposition 5.

Proposition 6. $\mathrm{R}_{d_{1}+d_{2}}^{e_{1}+e_{2}}\left(s_{1} \otimes s_{2}\right) \leq \mathrm{R}_{d_{1}}^{e_{1}}\left(s_{1}\right) \mathrm{R}_{d_{2}}^{e_{2}}\left(s_{2}\right)$

Proposition 7. Let $s$ be a k-tensor. Then $\mathrm{R}_{d}(s)=\mathrm{R}_{d}^{k d-d}(s)$.

The following theorem is our main technical result on which the rest of the paper rests. We note that for the tensor Kronecker product the statement is well-known in the context of algebraic complexity theory $[6,7,16,17,8]$.

Theorem 8. Let $t, s$ be $k$-tensors. If $t \unrhd^{e} s$ and $|\mathbb{F}| \geq e+2$, then we have $t \otimes \mathrm{T}_{e+1}(k) \geq s$.

Proof. By assumption there are matrices $A_{i}(\varepsilon)$ with entries polynomial in $\varepsilon$ such that

$$
\left(A_{1}(\varepsilon) \otimes \cdots \otimes A_{k}(\varepsilon)\right) t=\varepsilon^{d} s+\varepsilon^{d+1} s_{1}+\cdots+\varepsilon^{d+e} s_{e}
$$

for some tensors $s_{1}, \ldots, s_{e}$. Multiply both sides by $\varepsilon^{-d}$ and call the right-hand side $q(\varepsilon)$,

$$
\left(\varepsilon^{-d} A_{1}(\varepsilon) \otimes \cdots \otimes A_{k}(\varepsilon)\right) t=s+\varepsilon s_{1}+\cdots+\varepsilon^{e} s_{e}=: q(\varepsilon) .
$$

Let $\alpha_{0}, \ldots, \alpha_{e}$ be distinct nonzero elements of the ground field $\mathbb{F}$ (by assumption our ground field is large enough to do this). View $q(\varepsilon)$ as a polynomial in $\varepsilon$. Write $q(\varepsilon)$ as follows (Lagrange interpolation): 


$$
q(\varepsilon)=\sum_{j=0}^{e} q\left(\alpha_{j}\right) \prod_{\substack{0 \leq m \leq e: \\ m \neq j}} \frac{\varepsilon-\alpha_{m}}{\alpha_{j}-\alpha_{m}}
$$

We now see how to write $q(0)$ as a linear combination of the $q\left(\alpha_{j}\right)$, namely

$$
q(0)=\sum_{j=0}^{e} q\left(\alpha_{j}\right) \prod_{\substack{0 \leq m \leq e: \\ m \neq j}} \frac{\alpha_{m}}{\alpha_{m}-\alpha_{j}}
$$

that is,

$$
q(0)=\sum_{j=0}^{e} \beta_{j} q\left(\alpha_{j}\right) \quad \text { with } \quad \beta_{j}:=\prod_{\substack{0 \leq m \leq e: \\ m \neq j}} \frac{\alpha_{m}}{\alpha_{m}-\alpha_{j}} .
$$

Now we want to write $s$ as a restriction of $t \otimes \mathrm{T}_{e+1}(k)$. Define the linear maps $B_{1}:=$ $\sum_{j=0}^{e} \beta_{j} \alpha_{j}^{-d} A_{1}\left(\alpha_{j}\right) \otimes b_{j}^{*}$ and $B_{i}:=\sum_{j=0}^{e} \beta_{j} A_{i}\left(\alpha_{j}\right) \otimes b_{j}^{*}$ for $i \in\{2, \ldots, k\}$. Then $t \otimes$ $\mathrm{T}_{e+1}(k) \geq s$ because

$$
\begin{aligned}
\left(B_{1} \otimes \cdots \otimes B_{k}\right)\left(t \otimes \mathrm{T}_{e+1}(k)\right) & =\sum_{j=0}^{e} \beta_{j}\left(\alpha_{j}^{-d} A_{1}\left(\alpha_{j}\right) \otimes \cdots \otimes A_{k}\left(\alpha_{j}\right)\right) t \\
& =\sum_{j=0}^{e} \beta_{j} q\left(\alpha_{j}\right)=q(0)=s .
\end{aligned}
$$

This finishes the proof.

Remark 9. In the statement of Theorem 8 we assume that $|\mathbb{F}|$ is large enough. For small fields one can do the following. For $k, d \in \mathbb{N}$, let $[0 . . d]$ denote the set $\{0,1,2, \ldots, d\}$ and define the $k$-tensor

$$
\chi_{d}(k):=\sum_{\begin{array}{c}
a \in[0 . . d]^{k}: \\
a_{1}+\cdots+a_{k}=d
\end{array}} b_{a_{1}} \otimes \cdots \otimes b_{a_{k}} \in\left(\mathbb{F}^{d}\right)^{\otimes k} .
$$

Let $t, s$ be $k$-tensors. It is not hard to show that, if $t \unrhd_{d} s$, then $t \otimes \chi_{d}(k) \geq s$. By definition of $\chi_{d}(k)$ we have $\mathrm{R}\left(\chi_{d}(k)\right) \leq\left(\begin{array}{c}k+d-1 \\ k-1\end{array}\right)$. We may thus conclude that $t \nabla \mathrm{T}_{\left(\begin{array}{c}k+d-1 \\ k-1\end{array}\right)}(k) \geq s$.

We collect several almost immediate corollaries.

Corollary 10. Let $t_{i}, s_{i}$ be $k_{i}$-tensors for $i \in[n]$. Assume $\mathbb{F}$ is large enough.

1. If $\forall i: t_{i} \unrhd^{e_{i}} s_{i}$, then $\left(t_{1} \otimes \cdots \otimes t_{n}\right) \otimes \mathrm{T}_{\sum_{i} e_{i}+1}\left(\sum_{i} k_{i}\right) \geq s_{1} \otimes \cdots \otimes s_{n}$.

2. If $\forall i: t_{i} \unrhd_{d_{i}} s_{i}$, then $\left(t_{1} \otimes \cdots \otimes t_{n}\right) \otimes \mathrm{T}_{\sum_{i}\left(k_{i}-1\right) d_{i}+1}\left(\sum_{i} k_{i}\right) \geq s_{1} \otimes \cdots \otimes s_{n}$. 
Proof. To prove the first statement, apply Proposition 4 to obtain the degeneration $t_{1} \otimes \cdots \otimes t_{n} \unrhd^{\sum_{i} e_{i}} s_{1} \otimes \cdots \otimes s_{n}$. Theorem 8 yields the result. To prove the second statement, Proposition 5 gives $t_{i} \unrhd^{k_{i} d_{i}-d_{i}} s_{i}$. By Proposition $4, t_{1} \otimes \cdots \otimes t_{n} \unrhd^{\sum_{i} k_{i} d_{i}-d_{i}} s_{1} \otimes \cdots \otimes s_{n}$. Theorem 8 proves the statement.

Corollary 11. Let $s$ be a $k$-tensor. Assume $\mathbb{F}$ is large enough.

1. $\mathrm{R}\left(s^{\otimes n}\right) \leq(n e+1) \mathrm{R}^{e}(s)^{n}$.

2. $\mathrm{R}\left(s^{\otimes n}\right) \leq((k-1) n d+1) \mathrm{R}_{d}(s)^{n}$.

Proof. This follows from Corollary 10.

Corollary 12. Let $s$ be a $k$-tensor.

1. $\lim _{n \rightarrow \infty} \mathrm{R}\left(s^{\otimes n}\right)^{1 / n} \leq \underline{\mathrm{R}}(s)$.

2. $\lim _{n \rightarrow \infty} \mathrm{R}\left(s^{\otimes n}\right)^{1 / n}=\lim _{n \rightarrow \infty} \underline{\mathrm{R}}\left(s^{\otimes n}\right)^{1 / n}$.

3. If $\underline{\mathrm{R}}(s)<\mathrm{R}(s)$, then for some $n \in \mathbb{N}, \mathrm{R}\left(s^{\otimes n}\right)<\mathrm{R}(s)^{n}$.

\section{Tensor rank is not multiplicative under the tensor product}

Because of Corollary 11, in order to find nonmultiplicativity examples, it is enough to find a tensor $t$ for which $\mathrm{R}^{e}(t)<\mathrm{R}(t)$. We will give three families of examples of nonmultiplicativity. For $k \geq 3$, define the $k$-tensor

$$
W_{k}:=\sum_{\begin{array}{c}
i \in\{1,2\}^{k}: \\
\operatorname{type}(i)=(k-1,1)
\end{array}} b_{i_{1}} \otimes \cdots \otimes b_{i_{k}} \in\left(\mathbb{F}^{2}\right)^{\otimes k}
$$

where type $(i)=(k-1,1)$ means that $i$ is a permutation of $(1,1, \ldots, 1,2)$.

Proposition 13. Let $|\mathbb{F}|$ be large enough. Let $k \geq 3$. For $n$ large enough, we have a strict inequality $\mathrm{R}\left(W_{k}^{\otimes n}\right)<\mathrm{R}\left(W_{k}\right)^{n}$. For example, $\mathrm{R}\left(W_{3}^{\otimes 7}\right)<\mathrm{R}\left(W_{3}\right)^{7}$ and $\mathrm{R}\left(W_{8}^{\otimes 2}\right)<$ $\mathrm{R}\left(W_{8}\right)^{2}$.

Proof. The rank of $W_{k}$ equals $k$. This can be shown with the substitution method as explained in for example [18]. However, $\mathrm{R}^{k-1}\left(W_{k}\right) \leq 2$, namely

$$
\left(\left(\begin{array}{ll}
1 & 1 \\
\varepsilon & 0
\end{array}\right) \otimes \cdots \otimes\left(\begin{array}{ll}
1 & 1 \\
\varepsilon & 0
\end{array}\right) \otimes\left(\begin{array}{cc}
1 & -1 \\
\varepsilon & 0
\end{array}\right)\right) \mathrm{T}_{2}(k)=\varepsilon W_{k}+\varepsilon^{2}(\cdots)+\cdots+\varepsilon^{k}\left(b_{2} \otimes \cdots \otimes b_{2}\right) .
$$

Applying Corollary 11(1) to this degeneration gives $\mathrm{R}\left(W_{k}^{\otimes n}\right) \leq(n(k-1)+1) 2^{n}$. Therefore, for $n$ large enough, $\mathrm{R}\left(W_{k}^{\otimes n}\right) \leq 2^{n}(n(k-1)+1)<k^{n}=\mathrm{R}\left(W_{k}\right)^{n}$.

In fact, if $\operatorname{char}(\mathbb{F}) \neq 2$ and $\sqrt{2} \in \mathbb{F}$, then we can directly show a strict inequality for $n=2$ and $k=3$ as follows. 
Proposition 14. $\mathrm{R}\left(W_{3}^{\otimes 2}\right) \leq 8<9=\mathrm{R}\left(W_{3}\right)^{2}$ if char $\mathbb{F} \neq 2$ and $\sqrt{2} \in \mathbb{F}$.

Proof. As mentioned in the proof of Proposition 13, $\mathrm{R}\left(W_{3}\right)=3$. If $c \in \mathbb{F} \backslash\{0\}$ such that $\sqrt{c} \in \mathbb{F}$, then $\mathrm{R}\left(W_{3}+c b_{2} \otimes b_{2} \otimes b_{2}\right) \leq 2$. Namely,

$$
W_{3}+c b_{2} \otimes b_{2} \otimes b_{2}=\frac{1}{2 \sqrt{c}}\left(\left(b_{1}+\sqrt{c} b_{2}\right)^{\otimes 3}-\left(b_{1}-\sqrt{c} b_{2}\right)^{\otimes 3}\right) .
$$

(Over $\mathbb{C}$ this also follows from the fact that the Cayley hyperdeterminant evaluated at $W_{3}+c b_{2} \otimes b_{2} \otimes b_{2}$ is a nonzero constant times $c$. One may also see this by noting that the image of $W_{3}+c b_{2} \otimes b_{2} \otimes b_{2}$ under the moment map lies outside the image of the moment polytope associated to the orbit $\mathrm{GL}_{2} \times \mathrm{GL}_{2} \times \mathrm{GL}_{2} \cdot W[19,20]$.) We expand $W_{3} \otimes W_{3}$ as

$$
\begin{aligned}
W_{3} \otimes W_{3}=\left(W_{3}+b_{2} \otimes b_{2} \otimes b_{2}\right)^{\otimes 2} & -\left(W_{3}+\frac{1}{2} b_{2} \otimes b_{2} \otimes b_{2}\right) \otimes b_{2} \otimes b_{2} \otimes b_{2} \\
& -b_{2} \otimes b_{2} \otimes b_{2} \otimes\left(W_{3}+\frac{1}{2} b_{2} \otimes b_{2} \otimes b_{2}\right) .
\end{aligned}
$$

By the above, we know that the rank of $W_{3}+b_{2} \otimes b_{2} \otimes b_{2}$ and the rank of $W_{3}+\frac{1}{2} b_{2} \otimes b_{2} \otimes b_{2}$ are at most 2 . Therefore, the rank of $W_{3} \otimes W_{3}$ is at most $2^{2}+2+2=8$.

Remark 15. Let $S_{k}$ be the symmetric group of order $k$. Clearly the tensor $W_{3} \otimes W_{3}$ is invariant under the action of the subgroup $S_{3} \times S_{3} \subseteq S_{6}$ and under the action of the permutation $(14)(25)(36) \in S_{6}$ that swaps the two copies of $W_{3}$. Remarkably, the decomposition of $W_{3} \otimes W_{3}$ given in the proof of Proposition 14 also has this symmetry, in the sense that the above actions leave the set of simple terms appearing in the decomposition invariant. The decomposition is said to be partially symmetric. In fact, each term is itself invariant under $S_{3} \times S_{3}$.

Remark 16. It is stated in [21] that $\mathrm{R}\left(W_{3} \otimes W_{3}\right)=7$, which implies that $\mathrm{R}\left(W_{3} \otimes W_{3}\right)$ equals 7 or 8 . We obtained numerical evidence pointing to 8 . After the first version of our manuscript appeared on the arXiv, Chen and Friedland delivered a proof that $\mathrm{R}\left(W_{3} \otimes W_{3}\right) \geq 8$ [22]. For the third power, it is known that $\mathrm{R}\left(W_{3} \otimes W_{3} \otimes W_{3}\right)=16[23]$. A similar construction as in the proof of Proposition 14 gives $\mathrm{R}\left(W_{3} \otimes W_{3} \otimes W_{3}\right) \leq 21$. This upper bound is improved to 20 in [22].

In Proposition 13, we took the $n$th power of a tensor in $\left(\mathbb{F}^{2}\right)^{\otimes k}$ with $n$ large enough depending on $k$. In our next example, we take the square of a tensor in $\left(\mathbb{F}^{d}\right)^{\otimes k}$ with $d \geq 8$. For $k \geq 3$ and $q \geq 1$, define the tensor

$$
\operatorname{Str}_{q}^{k}:=\sum_{i=2}^{q+1} b_{i} \otimes b_{i} \otimes b_{1} \otimes b_{1}^{\otimes k-3}+b_{1} \otimes b_{i} \otimes b_{i} \otimes b_{1}^{\otimes k-3} \in\left(\mathbb{F}^{q+1}\right)^{\otimes k}
$$

This tensor is named after Strassen, who used $\operatorname{Str}_{q}^{3}$ to derive the upper bound $\omega \leq 2.48$ on the exponent of matrix multiplication $[14,18]$. 
Proposition 17. Assume that $\mathbb{F}$ is large enough. For $q \geq 7$ and any $k \geq 3$, we have a strict inequality $\mathrm{R}\left(\left(\operatorname{Str}_{q}^{k}\right)^{\otimes 2}\right)<\mathrm{R}\left(\operatorname{Str}_{q}^{k}\right)^{2}$.

Proof. The rank of $\operatorname{Str}_{q}^{k}$ equals $2 q$, again by the substitution method. We have $\mathrm{R}^{1}\left(\operatorname{Str}_{q}^{k}\right) \leq$ $q+1$, see the proof of Proposition 31 in [24]. Applying Corollary 11(1) to this degeneration gives $\mathrm{R}\left(\left(\operatorname{Str}_{q}^{k}\right)^{\otimes n}\right) \leq(n+1)(q+1)^{n}$. Therefore, for $q \geq 7$ and $n=2$, we have the strict inequality $\mathrm{R}\left(\left(\operatorname{Str}_{q}^{k}\right)^{\otimes 2}\right) \leq 3(q+1)^{2}<(2 q)^{2}=\mathrm{R}\left(\operatorname{Str}_{q}^{k}\right)^{2}$.

Our third example uses matrix multiplication tensors. Let $n_{1}, n_{2}, n_{3} \in \mathbb{N}$. Define the 3-tensor

$$
\begin{array}{r}
\left\langle n_{1}, n_{2}, n_{3}\right\rangle:=\sum_{i \in\left[n_{1}\right] \times\left[n_{2}\right] \times\left[n_{3}\right]}\left(b_{i_{1}} \otimes b_{i_{2}}\right) \otimes\left(b_{i_{2}} \otimes b_{i_{3}}\right) \otimes\left(b_{i_{3}} \otimes b_{i_{1}}\right) \\
\quad \in\left(\mathbb{F}^{n_{1}} \otimes \mathbb{F}^{n_{2}}\right) \otimes\left(\mathbb{F}^{n_{2}} \otimes \mathbb{F}^{n_{3}}\right) \otimes\left(\mathbb{F}^{n_{3}} \otimes \mathbb{F}^{n_{1}}\right) .
\end{array}
$$

Proposition 18. Assume that $\mathbb{F}$ is large enough. For $n \geq 78$, we have a strict inequality $\mathrm{R}\left(\langle 2,2,4\rangle^{\otimes n}\right)<\mathrm{R}(\langle 2,2,4\rangle)^{n}$.

Proof. The rank of $\langle 2,2,4\rangle$ equals 14 over any field [25, Theorem 2]. On the other hand, $\mathrm{R}^{4}(\langle 2,2,4\rangle) \leq 13$ over any field [26, Theorem 1]. Thus, when $\mathbb{F}$ is large enough Corollary 11(1) implies, for $n \geq 78$, the strict inequality $\mathrm{R}\left(\langle 2,2,4\rangle^{\otimes n}\right) \leq 13^{n}(4 n+1)<$ $14^{n}=\mathrm{R}(\langle 2,2,4\rangle)^{n}$.

In the language of graph tensors [8], Proposition 18 says that tensor rank is not multiplicative under taking disjoint unions of graphs.

\section{Generalized flattenings are multiplicative}

In the previous section we have seen that tensor rank can be strictly submultiplicative under the tensor product. We do not know whether the same is true for border rank. In fact, in this section we observe that lower bounds on border rank obtained from generalized flattenings are multiplicative. In this section we focus on 3-tensors for notational convenience. The ideas directly extend to $k$-tensors for any $k$.

Let $t$ be a tensor in $V_{1} \otimes V_{2} \otimes V_{3}$. We can transform $t$ into a matrix by grouping the tensor legs into two groups

$$
\begin{gathered}
V_{1} \otimes V_{2} \otimes V_{3} \rightarrow V_{1} \otimes\left(V_{2} \otimes V_{3}\right) \\
v_{1} \otimes v_{2} \otimes v_{3} \mapsto v_{1} \otimes\left(v_{2} \otimes v_{3}\right) .
\end{gathered}
$$

(There are three ways to do this for a 3 -tensor.) This is called flattening. The rank of a flattening of $t$ is a lower bound for the border rank of $t$. (Rank and border rank are equal for matrices.) 
We now define generalized flattenings. Let $t$ be a tensor in $V_{1} \otimes V_{2} \otimes V_{3}$. Instead of a basic flattening $V_{1} \otimes V_{2} \otimes V_{3} \rightarrow V_{1} \otimes\left(V_{2} \otimes V_{3}\right)$, we choose vector spaces $V_{1}^{\prime}$ and $V_{2}^{\prime}$ and apply some linear map $F: V_{1} \otimes V_{2} \otimes V_{3} \rightarrow V_{1}^{\prime} \otimes V_{2}^{\prime}$ to $t$. To obtain a border rank lower bound using $F$ we have to compensate for the fact that $F$ possibly increases the border rank of a simple tensor. The following lemma describes the resulting lower bound.

Lemma 19. Let $t \in V_{1} \otimes V_{2} \otimes V_{3}$ be a tensor. Let

$$
F: V_{1} \otimes V_{2} \otimes V_{3} \rightarrow V_{1}^{\prime} \otimes V_{2}^{\prime}
$$

be a linear map. The border rank of $t$ is at least

$$
\underline{\mathrm{R}}(t) \geq \frac{\mathrm{R}(F(t))}{\max \mathrm{R}\left(F\left(v_{1} \otimes v_{2} \otimes v_{3}\right)\right)},
$$

where the maximum is over all simple tensors $v_{1} \otimes v_{2} \otimes v_{3}$ in $V_{1} \otimes V_{2} \otimes V_{3}$.

Proof. Suppose $\underline{\mathrm{R}}(t)=r$. Then there is a sequence of tensors $t_{i}$ converging to $t$ with $\mathrm{R}\left(t_{i}\right) \leq r$ for each $i$. Each $t_{i}$ thus has a decomposition into simple tensors $t_{i}=\sum_{j=1}^{r} t_{i, j}$. Since $F\left(t_{i}\right) \rightarrow F(t)$, there exists an $i_{0}$ such that for all $i \geq i_{0}$ we have $\mathrm{R}\left(F\left(t_{i}\right)\right) \geq \mathrm{R}(F(t))$. Moreover, we have the inequalities $\mathrm{R}\left(F\left(t_{i}\right)\right) \leq \sum_{j=1}^{r} \mathrm{R}\left(F\left(t_{i, j}\right)\right) \leq$ $r \cdot \max _{s} \mathrm{R}(F(s))$, where the maximum is over all simple tensors $s$. We conclude that $\underline{\mathrm{R}}(t) \geq \mathrm{R}(F(t)) / \max _{s} \mathrm{R}(F(s))$.

Note that the right-hand side of (2) might not be an integer. The lower bound in (2) is multiplicative under the tensor product in the following sense.

Proposition 20. Let $s \in V_{1} \otimes V_{2} \otimes V_{3}$ and $t \in W_{1} \otimes W_{2} \otimes W_{3}$ be tensors. Let $F_{1}$ : $V_{1} \otimes V_{2} \otimes V_{3} \rightarrow V_{1}^{\prime} \otimes V_{2}^{\prime}$ and $F_{2}: W_{1} \otimes W_{2} \otimes W_{3} \rightarrow W_{1}^{\prime} \otimes W_{2}^{\prime}$ be linear maps. The border rank of $s \otimes t \in V_{1} \otimes V_{2} \otimes V_{3} \otimes W_{1} \otimes W_{2} \otimes W_{3}$ is at least

$$
\underline{\mathrm{R}}(s \otimes t) \geq \frac{\mathrm{R}\left(F_{1}(s)\right)}{\max \mathrm{R}\left(F_{1}\left(v_{1} \otimes v_{2} \otimes v_{3}\right)\right)} \frac{\mathrm{R}\left(F_{2}(t)\right)}{\max \mathrm{R}\left(F_{2}\left(w_{1} \otimes w_{2} \otimes w_{3}\right)\right)}
$$

where the maximizations are over simple tensors in $V_{1} \otimes V_{2} \otimes V_{3}$ and in $W_{1} \otimes W_{2} \otimes W_{3}$ respectively.

Proof. Combine $F_{1}$ and $F_{2}$ into a single linear map

$$
F: V_{1} \otimes V_{2} \otimes V_{3} \otimes W_{1} \otimes W_{2} \otimes W_{3} \rightarrow\left(V_{1}^{\prime} \otimes W_{1}^{\prime}\right) \otimes\left(V_{2}^{\prime} \otimes W_{2}^{\prime}\right)
$$

One then follows the proof of Lemma 19 and uses the fact that matrix rank is multiplicative under the tensor Kronecker product. 
Young flattenings $[27,28]$ are a special case of generalized flattenings. For completeness, we finish with a concise description of Young flattenings and the corresponding multiplicativity statement. We work over the complex numbers $\mathbb{C}$. Let $S_{\lambda} V$ be an irreducible $\mathrm{GL}_{V}$-module of type $\lambda$. Consider the space $V \otimes S_{\lambda} V$ as a $\mathrm{GL}_{V}$-module under the diagonal action. The Pieri rule says that we have a $\mathrm{GL}_{V}$-decomposition

$$
V \otimes S_{\lambda} V \cong \bigoplus_{\mu} S_{\mu} V
$$

where the direct sum is over partitions $\mu$ of length at most $\operatorname{dim} V$ obtained from $\lambda$ by adding a box in the Young diagram of $\lambda$. This decomposition yields GL $_{V}$-equivariant embeddings $S_{\mu} V \hookrightarrow V \otimes S_{\lambda} V$, called Pieri inclusions or partial polarization maps. These maps are unique up to scaling. Such a Pieri inclusion corresponds to a $\mathrm{GL}_{V}$-equivariant map $\phi_{\mu, \lambda}: V^{*} \rightarrow S_{\mu} V^{*} \otimes S_{\lambda} V$. Every element $\phi_{\mu, \lambda}(v)$ is called a Pieri map. The Young flattening $F_{\mu, \lambda}$ on $V_{1} \otimes V_{2}^{*} \otimes V_{3}$ is obtained by first applying the map $\phi_{\mu, \lambda}$ to one tensor leg,

$$
V_{1} \otimes V_{2}^{*} \otimes V_{3} \rightarrow V_{1} \otimes S_{\mu} V_{2}^{*} \otimes S_{\lambda} V_{2} \otimes V_{3}
$$

and then flattening into a matrix,

$$
V_{1} \otimes S_{\mu} V_{2}^{*} \otimes S_{\lambda} V_{2} \otimes V_{3} \rightarrow\left(V_{1} \otimes S_{\mu} V_{2}^{*}\right) \otimes\left(S_{\lambda} V_{2} \otimes V_{3}\right)
$$

Note that for any simple tensor $v_{1} \otimes v_{2} \otimes v_{3}$, the rank of $F_{\mu, \lambda}\left(v_{1} \otimes v_{2} \otimes v_{3}\right)$ equals the rank of $\phi_{\mu, \lambda}\left(v_{2}\right)$. Proposition 20 thus specializes as follows.

Proposition 21. Let $s \in V_{1} \otimes V_{2} \otimes V_{3}$ and $t \in W_{1} \otimes W_{2} \otimes W_{3}$. Let $\lambda, \mu$ and $\nu, \kappa$ be pairs of partitions as above. The border rank of $s \otimes t \in V_{1} \otimes V_{2} \otimes V_{3} \otimes W_{1} \otimes W_{2} \otimes W_{3}$ is at least

$$
\underline{\mathrm{R}}(s \otimes t) \geq \frac{\mathrm{R}\left(F_{\mu, \lambda}(s)\right)}{\max \mathrm{R}\left(\phi_{\mu, \lambda}\left(v_{2}\right)\right)} \frac{\mathrm{R}\left(F_{\nu, \kappa}(t)\right)}{\max \mathrm{R}\left(\phi_{\nu, \kappa}\left(w_{2}\right)\right)}
$$

where the maximizations are over $v_{2} \in V_{2}$ and $w_{2} \in W_{2}$ respectively.

We refer to [29] for an overview of the applications of Young flattenings.

\section{Multiplicativity for complex matrix pencils and 2-tensors}

In this section all vector spaces are over the complex numbers. The goal of this section is to prove the following proposition.

Proposition 22. Let $s \in \mathbb{C} \otimes \mathbb{C}^{d} \otimes \mathbb{C}^{d}$ and $t \in \mathbb{C}^{2} \otimes \mathbb{C}^{n} \otimes \mathbb{C}^{m}$. Then

$$
R(t \otimes s)=R(t \otimes s)=R(t) R(s)
$$


Remark 23. Proposition 22 shows that Example 2 is essentially minimal over the complex numbers. Namely, any example of non-multiplicativity of tensor rank under $\otimes$ must either be with a 5 -tensor in $\left(\mathbb{C}^{d} \otimes \mathbb{C}^{d}\right) \otimes\left(\mathbb{C}^{d_{1}} \otimes \mathbb{C}^{d_{2}} \otimes \mathbb{C}^{d_{3}}\right)$ with $d_{1}, d_{2}, d_{3} \geq 3, d \geq 2$ or in a tensor space of order 6 or more. Moreover, one can show using Proposition 22 and the well-known classification of the $\mathrm{GL}_{2}^{\times 3}$-orbits in $\mathbb{C}^{2} \otimes \mathbb{C}^{2} \otimes \mathbb{C}^{2}$ that if $s, t \in \mathbb{C}^{2} \otimes \mathbb{C}^{2} \otimes \mathbb{C}^{2}$ and $\mathrm{R}(s \otimes t)<\mathrm{R}(s) \mathrm{R}(t)$, then $s$ and $t$ are both isomorphic to the tensor $W_{3}$.

The elements of $\mathbb{C}^{2} \otimes \mathbb{C}^{n} \otimes \mathbb{C}^{m}$ are often called matrix pencils. The tensor rank of matrix pencils is completely understood, in the sense that every matrix pencil is equivalent under local isomorphisms to a pencil in canonical form, for which the rank is given by a simple formula. This formula will allow us to give a short proof of Proposition 22.

We begin with introducing the canonical form for matrix pencils. For a proof we refer to $\left[30\right.$, Chapter XII]. Recall that the standard basis elements of $\mathbb{C}^{n}$ are denoted by $b_{1}, \ldots, b_{n}$.

Definition 24. Given $t_{i} \in U \otimes V_{i} \otimes W_{i}$, define $\operatorname{diag}_{U}\left(t_{1}, \ldots, t_{n}\right)$ as the image of $\bigoplus_{i=1}^{n} t_{i}$ under the natural inclusion $\bigoplus_{i}\left(U \otimes V_{i} \otimes W_{i}\right) \rightarrow U \otimes\left(\bigoplus_{i} V_{i}\right) \otimes\left(\bigoplus_{i} W_{i}\right)$. For $\varepsilon \in \mathbb{N}$ define the tensor $L_{\varepsilon} \in \mathbb{C}^{2} \otimes \mathbb{C}^{\varepsilon} \otimes \mathbb{C}^{\varepsilon+1}$ by

$$
\begin{aligned}
& L_{\varepsilon}:=b_{1} \otimes \sum_{i=1}^{\varepsilon} b_{i} \otimes b_{i}+b_{2} \otimes \sum_{i=1}^{\varepsilon} b_{i} \otimes b_{i+1}
\end{aligned}
$$

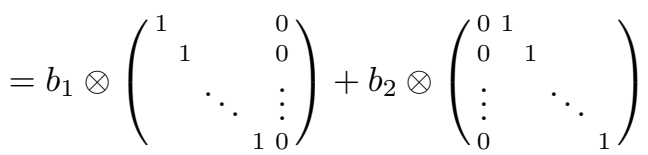

and for $\eta \in \mathbb{N}$ define the tensor $N_{\eta} \in \mathbb{C}^{2} \otimes \mathbb{C}^{\eta+1} \otimes \mathbb{C}^{\eta}$ by

$$
\begin{aligned}
N_{\eta} & :=b_{1} \otimes \sum_{i=1}^{\eta} b_{i} \otimes b_{i}+b_{2} \otimes \sum_{i=1}^{\eta} b_{i+1} \otimes b_{i} \\
& =b_{1} \otimes\left(\begin{array}{cccc}
1 & & & \\
& 1 & & \\
& & \ddots & \\
& & & \\
& 0 & \cdots & 0
\end{array}\right)+b_{2} \otimes\left(\begin{array}{cccc}
0 & 0 & \cdots & 0 \\
1 & & & \\
& 1 & & \\
& & \ddots & \\
& & & 1
\end{array}\right) .
\end{aligned}
$$

Theorem 25 (Canonical form). Let $t \in \mathbb{C}^{2} \otimes \mathbb{C}^{n} \otimes \mathbb{C}^{m}$. There exist invertible linear maps $A \in \mathrm{GL}_{2}, B \in \mathrm{GL}_{n}$ and $C \in \mathrm{GL}_{m}$ and natural numbers $\varepsilon_{1}, \ldots, \varepsilon_{p}, \eta_{1}, \ldots, \eta_{q} \in \mathbb{N}$ and an $\ell \times \ell$ Jordan matrix $F$ such that, with $M=b_{1} \otimes I_{\ell}+b_{2} \otimes F$, we have

$$
(A \otimes B \otimes C) t=\operatorname{diag}_{\mathbb{C}^{2}}\left(0, L_{\varepsilon_{1}}, \ldots, L_{\varepsilon_{p}}, N_{\eta_{1}}, \ldots, N_{\eta_{q}}, M\right)
$$

where the 0 stands for some 0-tensor of appropriate dimensions. The right-hand side of (3) is called the canonical form of $t$. 
Next we give a formula for the tensor rank of matrix pencils in canonical form (Theorem 27). Theorem 27 is due to Grigoriev [31], JáJá [32] and Teichert [33], see also [5, Theorem 19.4] or [29, Theorem 3.11.1.1].

Definition 26. Let $F$ be a Jordan matrix with eigenvalues $\lambda_{1}, \lambda_{2}, \ldots, \lambda_{p}$. Let $d\left(\lambda_{i}\right)$ be the number of Jordan blocks in $F$ of size at least two with eigenvalue $\lambda_{i}$. Define $m(F):=$ $\max _{i} d\left(\lambda_{i}\right)$.

Theorem 27. Let $t=\operatorname{diag}_{\mathbb{C}^{2}}\left(0, L_{\varepsilon_{1}}, \ldots, L_{\varepsilon_{p}}, N_{\eta_{1}}, \ldots, N_{\eta_{q}}, b_{1} \otimes I_{\ell}+b_{2} \otimes F\right)$ be a tensor in canonical form as in (3). The tensor rank of $t$ equals

$$
\mathrm{R}(t)=\sum_{i=1}^{p}\left(\varepsilon_{i}+1\right)+\sum_{i=1}^{q}\left(\eta_{i}+1\right)+\ell+m(F) .
$$

Example 28. Let $W_{3}=b_{2} \otimes b_{1} \otimes b_{1}+b_{1} \otimes b_{2} \otimes b_{1}+b_{1} \otimes b_{1} \otimes b_{2} \in\left(\mathbb{C}^{2}\right)^{\otimes 3}$ as in Example 2 . The canonical form of $W_{3}$ is

$$
W_{3} \cong b_{1} \otimes\left(\begin{array}{ll}
1 & 0 \\
0 & 1
\end{array}\right)+b_{2} \otimes\left(\begin{array}{ll}
0 & 1 \\
0 & 0
\end{array}\right)
$$

so in the notation of Theorem 25 we have $p=q=0$ and $F=\left(\begin{array}{ll}0 & 1 \\ 0 & 0\end{array}\right)$. We can thus apply Theorem 27 with $\ell=2$ and $m(F)=1$ to get $\mathrm{R}\left(W_{3}\right)=2+1=3$.

We are now ready to give the short proof of Proposition 22.

Proof of Proposition 22. Let $s \in \mathbb{C} \otimes \mathbb{C}^{d} \otimes \mathbb{C}^{d}, t \in \mathbb{C}^{2} \otimes \mathbb{C}^{n} \otimes \mathbb{C}^{m}$. We may assume that $s=1 \otimes \sum_{i=1}^{r} b_{i} \otimes b_{i}$ with $r=\mathrm{R}(s)$. By Theorem 25 we may assume that $t$ is in canonical form, $t=\operatorname{diag}_{\mathbb{C}^{2}}\left(0, L_{\varepsilon_{1}}, \ldots, L_{\varepsilon_{p}}, N_{\eta_{1}}, \ldots, N_{\eta_{q}}, M\right)$. The tensor Kronecker product $t \otimes s$ is isomorphic to

$$
t \otimes s \cong \operatorname{diag}_{\mathbb{C}^{2}}(\underbrace{t, \ldots, t}_{r})
$$

By an appropriate local basis transformation we put this in canonical form

$$
t \otimes s \cong \operatorname{diag}_{\mathbb{C}^{2}}\left(L_{\varepsilon_{1}}^{\oplus r}, \ldots, L_{\varepsilon_{p}}^{\oplus r}, N_{\eta_{1}}^{\oplus r}, \ldots, N_{\eta_{q}}^{\oplus r}, M^{\oplus r}\right)
$$

which by Theorem 27 has rank $r \cdot \mathrm{R}(t)=\mathrm{R}(s) \mathrm{R}(t)$.

Remark 29. Proposition 22 is also true over the finite field $\mathbb{F}_{q}$ when $q \geq n, m$. To see this one may use the formula from [5, Section 19.5] for the rank of pencils over finite fields, which for $q \geq n, m$ is as follows: 


$$
\mathrm{R}(t)=\sum_{i=1}^{p}\left(\varepsilon_{i}+1\right)+\sum_{i=1}^{q}\left(\eta_{i}+1\right)+\ell+\delta(B) .
$$

Here $B$ is the regular part of the pencil $t$ and $\delta(B)$ is the number of invariant divisors of $B$ that do not decompose into a product of unassociated linear factors. (We refer to [5] for definitions.) The invariant divisors of $\operatorname{diag}(B, \ldots, B)$ are just the invariant divisors of $B$ counted for each copy of $B$ and so Proposition 22 follows.

We note that part of the results in this section have been independently obtained in Section 2 of [22].

\section{Acknowledgements}

We thank Jonathan Skowera for discussion, Fulvio Gesmundo for suggestions regarding Section 4, and Nick Vannieuwenhoven for discussion regarding the literature. We acknowledge financial support from the European Research Council (ERC Grant Agreement no. 337603), the Danish Council for Independent Research (Sapere Aude), and VILLUM FONDEN via the QMATH Centre of Excellence (Grant no. 10059). JZ is supported by NWO (617.023.116) and the QuSoft Research Center for Quantum Software.

\section{References}

[1] V. Strassen, The asymptotic spectrum of tensors, J. Reine Angew. Math. 384 (1988) 102-152, https://doi.org/10.1515/crll.1988.384.102.

[2] J. Draisma, Multilinear Algebra and Applications (lecture notes), URL https://mathsites.unibe.ch/ jdraisma/publications/mlappl.pdf, 2015.

[3] R. Saptharishi, A survey of lower bounds in arithmetic circuit complexity 3.0.2, online survey, https://github.com/dasarpmar/lowerbounds-survey, 2016.

[4] V. de Silva, L.-H. Lim, Tensor rank and the ill-posedness of the best low-rank approximation problem, SIAM J. Matrix Anal. Appl. 30 (3) (2008) 1084-1127, https://doi.org/10.1137/06066518X.

[5] P. Bürgisser, M. Clausen, M.A. Shokrollahi, Algebraic Complexity Theory, Grundlehren Math. Wiss., vol. 315, Springer-Verlag, Berlin, 1997, https://doi.org/10.1007/978-3-662-03338-8.

[6] D. Bini, G. Lotti, F. Romani, Approximate solutions for the bilinear form computational problem, SIAM J. Comput. 9 (4) (1980) 692-697, https://doi.org/10.1137/0209053.

[7] D. Bini, Relations between exact and approximate bilinear algorithms. Applications, Calcolo 17 (1) (1980) 87-97, https://doi.org/10.1007/BF02575865.

[8] M. Christandl, P. Vrana, J. Zuiddam, Asymptotic tensor rank of graph tensors: beyond matrix multiplication, arXiv:1609.07476, 2016.

[9] V. Strassen, Gaussian elimination is not optimal, Numer. Math. 13 (4) (1969) 354-356, https:// doi.org/10.1007/BF02165411.

[10] S. Winograd, On multiplication of $2 \times 2$ matrices, Linear Algebra Appl. 4 (4) (1971) 381-388, https://doi.org/10.1016/0024-3795(71)90009-7.

[11] J.M. Landsberg, The border rank of the multiplication of $2 \times 2$ matrices is seven, J. Amer. Math. Soc. 19 (2) (2006) 447-459, https://doi.org/10.1090/S0894-0347-05-00506-0.

[12] M. Bläser, M. Christandl, J. Zuiddam, The border support rank of two-by-two matrix multiplication is seven, arXiv:1705.09652, 2017.

[13] A. Alder, Grenzrang und Grenzkomplexität aus algebraischer und topologischer Sicht, Ph.D. thesis, Zürich University, 1984.

[14] V. Strassen, Relative bilinear complexity and matrix multiplication, J. Reine Angew. Math. $375 / 376$ (1987) 406-443, https://doi.org/10.1515/crll.1987.375-376.406. 
[15] T. Lehmkuhl, T. Lickteig, On the order of approximation in approximative triadic decompositions of tensors, Theoret. Comput. Sci. 66 (1) (1989) 1-14, https://doi.org/10.1016/0304-3975(89)90141-2.

[16] A. Schönhage, Partial and total matrix multiplication, SIAM J. Comput. 10 (3) (1981) 434-455, https://doi.org/10.1137/0210032.

[17] V. Strassen, Degeneration and complexity of bilinear maps: some asymptotic spectra, J. Reine Angew. Math. 413 (1991) 127-180, https://doi.org/10.1515/crll.1991.413.127.

[18] M. Bläser, Fast matrix multiplication, Grad. Surv., Theory Comput. Libr. 5 (2013), https://doi.org/ 10.4086/toc.gs.2013.005.

[19] M. Walter, B. Doran, D. Gross, M. Christandl, Entanglement polytopes: multiparticle entanglement from single-particle information, Science 340 (6137) (2013) 1205-1208, https://doi.org/10.1126/ science.1232957.

[20] A. Sawicki, M. Oszmaniec, M. Kuś, Convexity of momentum map, Morse index, and quantum entanglement, Rev. Math. Phys. 26 (3) (2014) 1450004, 39, https://doi.org/10.1142/S0129055X14500044.

[21] N. Yu, E. Chitambar, C. Guo, R. Duan, Tensor rank of the tripartite state W tensor $n$, Phys. Rev. A 81 (1) (2010) 014301, https://doi.org/10.1103/PhysRevA.81.014301.

[22] L. Chen, S. Friedland, The tensor rank of tensor product of two three-qubit W states is eight, arXiv:1708.08578, 2017.

[23] J. Zuiddam, A note on the gap between rank and border rank, Linear Algebra Appl. 525 (2017) 33-44, https://doi.org/10.1016/j.laa.2017.03.015.

[24] H. Buhrman, M. Christandl, J. Zuiddam, Nondeterministic quantum communication complexity: the cyclic equality game and iterated matrix multiplication, in: 8th Innovations in Theoretical Computer Science Conference (ITCS 2017), 2017, pp. 24:1-24:18, arXiv:1603.03757, https://doi.org/ 10.4230/LIPIcs.ITCS.2017.24.

[25] J.E. Hopcroft, L.R. Kerr, On minimizing the number of multiplications necessary for matrix multiplication, SIAM J. Appl. Math. 20 (1971) 30-36, https://doi.org/10.1137/0120004.

[26] V.B. Alekseev, A.V. Smirnov, On the exact and approximate bilinear complexities of multiplication of $4 \times 2$ and $2 \times 2$ matrices, Proc. Steklov Inst. Math. 282 (suppl. 1) (2013) S123-S139, https://doi.org/10.1134/S0081543813070079.

[27] V. Strassen, Rank and optimal computation of generic tensors, Linear Algebra Appl. 52/53 (1983) 645-685, https://doi.org/10.1016/0024-3795(83)80041-X.

[28] J.M. Landsberg, G. Ottaviani, New lower bounds for the border rank of matrix multiplication, Theory Comput. 11 (2015) 285-298, https://doi.org/10.4086/toc.2015.v011a011, arXiv:1112.6007.

[29] J.M. Landsberg, Tensors: Geometry and Applications, vol. 128, American Mathematical Society, Providence, RI, USA, 2012.

[30] F.R. Gantmacher, The Theory of Matrices, vol. 2. Transl. from the Russian by K.A. Hirsch. Reprint of the 1959 translation, AMS Chelsea Publishing, Providence, RI, 1998.

[31] D.Y. Grigoriev, Multiplicative complexity of a pair of bilinear forms and of the polynomial multiplication, in: Mathematical Foundations of Computer Science, 1978, Proc. Seventh Sympos., Zakopane, 1978, in: Lecture Notes in Comput. Sci., vol. 64, Springer, Berlin, New York, 1978, pp. 250-256, https://doi.org/10.1007/3-540-08921-7_72.

[32] J. JáJá, Optimal evaluation of pairs of bilinear forms, SIAM J. Comput. 8 (3) (1979) 443-462, https://doi.org/10.1137/0208037.

[33] L. Teichert, Die Komplexität von Bilinearformpaaren über beliebigen Körpern, MathematischNaturwissenschaftliche Fakultät der Technischen Universität Clausthal, 1986. 